\title{
Technè
}

La science au service de l'histoire de l'art et de la préservation des biens culturels

44 | 2016

Archives de l'humanité : les restes humains patrimonialisés

\section{Gestion et étude des restes humains en fluides}

Les collections du Muséum national d'histoire naturelle de Paris : histoire, enjeux et valorisations

Management and study of human remains in fluids. The collections of the Muséum National d'Histoire Naturelle, Paris: history, challenges and developments

\section{Marc Herbin et Jacques Cuisin}

\section{(2) OpenEdition}

12 Journals

\section{Édition électronique}

URL : http://journals.openedition.org/techne/977

DOI : 10.4000/techne.977

ISSN : 2534-5168

Éditeur

C2RMF

\section{Édition imprimée}

Date de publication : 1 novembre 2016

Pagination : 33-36

ISBN : 978-2-7118-6339-6

ISSN : 1254-7867

Référence électronique

Marc Herbin et Jacques Cuisin, "Gestion et étude des restes humains en fluides », Technè [En ligne], 44 | 2016, mis en ligne le 19 décembre 2019, consulté le 28 juillet 2020. URL : http:// journals.openedition.org/techne/977 ; DOI : https://doi.org/10.4000/techne.977

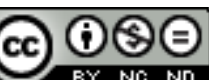

La revue Technè. La science au service de l'histoire de l'art et de la préservation des biens culturels est mise à disposition selon les termes de la Licence Creative Commons Attribution - Pas d'Utilisation Commerciale - Pas de Modification 4.0 International. 
Marc Herbin

Jacques Cuisin

\section{Gestion et étude des restes humains en fluides. Les collections du Muséum national d'histoire naturelle de Paris : histoire, enjeux et valorisations}

Management and study of human remains in fluids. The collections of the Muséum National d'Histoire Naturelle, Paris: history, challenges and developments
Résumé. Les restes humains sont présents dans les collections $d u$ Muséum national d'histoire naturelle de Paris depuis sa création. Depuis toujours, ces pièces ont constitué un support pour l'enseignement, un matériel essentiel pour la recherche et, bien sûr, des pièces incontournables dans les présentations muséales. Par conséquent, une attention particulière doit être portée à ces collections, le bien-fondé d'une collection étant qu'elle doit servir pour être pleinement valorisée.

Mots-clés. Anatomie comparée, conservation, recherche, muséologie, déontologie.

\begin{abstract}
Human remains have been part of the collections of the Muséum National d'Histoire Naturelle, Paris, since it was founded. These items have always been used for teaching purposes, provided basic material for research, and, of course, been put on display in the museum. Consequently, particular attention must be paid to these collections, the legitimacy of a collection being that its potential should be fully exploited.
\end{abstract}

Keywords. Comparative anatomy, conservation, research, museology, deontology.
«L'anatomie comparée est le lieu pour l'étude et la comparaison de l'homme, des autres animaux fossiles et actuels », Henri Ducrotay de Blainville, directeur de la chaire d'Anatomie comparée (1832-1850).

\section{Introduction}

Les collections de restes humains en fluides du Muséum national d'histoire naturelle sont issues des premières collections des différents cabinets du XVIII ${ }^{\mathrm{e}}$ siècle (Cabinet dit « du Roi », saisies révolutionnaires, jusqu'en 1793, émergence du Muséum d'histoire naturelle...). Ces restes humains en fluides, regroupés initialement dans la chaire d'Anatomie des animaux seront, au cours du XIX ${ }^{\mathrm{e}}$ siècle, répartis dans les chaires d'Anatomie comparée (initialement «Anatomie des animaux ») et d'Anatomie humaine (future chaire d'Anthropologie). Les premières collections étaient conservées dans diverses solutions à base d'alcools forts ${ }^{1}$, puis, avec la découverte des propriétés du formaldéhyde dès 1859, le formol (solution aqueuse de cet aldéhyde) remplace les liquides auparavant employés ${ }^{2}$, alors que l'on continue à utiliser l'alcool à $70 \%$ en Anthropologie.

Le fait de choisir tel ou tel type de liquide conservateur relève aujourd'hui essentiellement des modes de valorisation des collections. Si, dans l'une, on utilise uniquement la collection à des fins de présentation au public ou d'enseignement, dans l'autre en revanche, l'utilisation du formol permet de garantir les propriétés physiologiques des tissus lors des investigations propres aux études d'anatomie comparative. Ce type de fluide conservateur permet aujourd'hui encore d'apporter à la communauté scientifique un corpus de données adapté aux investigations macro- et microscopiques par le biais de techniques diverses ${ }^{3}$. Cette capacité confère toute leur spécificité aux collections d'anatomie comparée en fluides en ce sens que les spécimens sont, au moins partiellement, fongibles puisqu'ils peuvent toujours faire l'objet de prélèvements destructeurs et/ou être disséqués. Sur un plan matériel, en effet, ces collections s'inscrivent parfaitement dans un schéma technique mis en œuvre pour d'autres mammifères, par exemple.

À cela s'ajoute une composante déontologique forte, puisque le corps humain, sous quelque forme que ce soit, ne saurait être traité à l'instar d'autres organismes du règne animal.

Toutes ces particularités font que les collections de restes humains en fluides doivent être gérées différemment des autres collections classiques, au moins pour ce qui relève de leur valorisation, mais aussi pour ce qui relève de leur préservation, même en contexte muséal. 


\section{Origines}

La technique de la collection en fluides a initialement été mise au point par et pour les anatomistes, car dès le $\mathrm{XVIII}^{\mathrm{e}}$ siècle, voire auparavant, médecins, apothicaires et chirurgiens ont eu besoin de conserver des corps à des fins d'exemple et d'enseignement, et ont essayé de nombreux procédés dans le but de pouvoir préserver le plus longtemps possible les corps ou les parties anatomiques jugées dignes d'intérêt dans une démarche de connaissance. En effet, pour conserver un corps, il faut ralentir ou, mieux, stopper la décomposition des tissus, tout en évitant les contaminations bactériennes. Ambroise Paré (vers 1510-1590) vante le premier les propriétés « conservatrices » de l'alcool (esprit de vin), mais celles-ci ne sont pas assez efficaces pour préserver les tissus de manière durable et intègre. Frederick Ruysch (16791717) franchit une étape décisive en mélangeant de nombreux constituants, solides et liquides, connus pour leurs pouvoirs intrinsèques conservateurs et/ou aseptiques. Ce n'est cependant que la découverte du formaldéhyde et de ses propriétés fixatrices et conservatrices, entre 1863 et 1910, qui va permettre de vraiment stopper l'autolyse des tissus et de constituer une barrière efficace aux divers agents contaminants et dégradants. Ainsi si, aujourd'hui encore, seules les solutions à base d'aldéhydes sont adaptées à la fixation pérenne des tissus organiques, il existe en revanche trois familles de solutions conservatrices : les alcooliques, celles à base d'aldéhydes, et les solutions mixtes.

Au Muséum national, depuis Daubenton (Cabinet du Roi), les restes humains en fluides sont en grande partie intégrés à la collection d'Anatomie comparée, et cela dans la droite ligne des anatomistes médecins et chirurgiens des $\mathrm{XVII}^{\mathrm{e}}$-XVIII ${ }^{\mathrm{e}}$ siècles. En revanche, la plupart des restes humains secs (squelettes ou autres) relèvent des collections d'Anthropologie, chaire existante dès l'origine du Muséum (nommée chaire d'Anatomie humaine en 1793 et rebaptisée Anthropologie en 1855). L'ensemble des collections d'Anatomie comparée regroupe tous les taxons de vertébrés, l'Homme étant, très spécialement depuis Buffon, étroitement associé aux Primates. Ces restes humains en fluides sont pour la plupart conservés dans une solution de formol à $10 \%$. Cependant, quelques spécimens sont conservés dans d'autres solutions, telles le liquide de Bouin. D'autres restes plus anciens sont encore dans leur solution conservatrice initiale (alcool, et autres solutions complexes en cours d'identification). Le Muséum national ayant longtemps donné le ton en matière de technique, on retrouve peu ou prou les mêmes solutions dans d'autres collections françaises comparables. Dans une grande partie de l'Europe jusqu'à la moitié du $\mathrm{XX}^{\mathrm{e}}$ siècle environ, le formol a eu la préférence des anatomistes et des médecins en tant que liquide à la fois fixatif et préservatif. Aujourd'hui, pour des questions d'Hygiène et Sécurité, couplées à des normes de composition de l'air ambiant de plus en plus drastiques, les collections présentées au public tendent à être préservées en solution dite de Kaïserling (et plus précisément, celle sans formol, dite « Kaïserling III modifiée »), ce qui suppose tout de même une fixation préalable irréprochable.

\section{Modalités}

Outre la qualité du fluide préservatif, les contenants ont également une grande importance, car ils garantissent la pérennité de l'ensemble spécimen-fluide. Ils sont de natures et de formes très variables selon les époques, les types de collections et les types de musées. Entre les XVII ${ }^{\mathrm{e}}$ et XVIII ${ }^{\mathrm{e}}$ siècles, on ne différencie pas les contenants destinés aux restes humains de ceux destinés aux autres collections de naturalia, car toutes les présentations visent à émerveiller les publics, dans une mise en scène digne des cabinets de curiosités de la Renaissance, même si la scénographie des spécimens et pièces d'anatomie comparée ne connaît un essor réel et une dimension particulière qu'au XIX ${ }^{\mathrm{e}}$ siècle $^{4}$. À la fin de ce siècle, les collections médicales ou anatomiques adaptent les contenants aux pièces présentées, afin qu'elles soient le plus didactiques possible (fig. 1). Il s'agit de faire passer un discours scientifique ou médical (fig. 2) ; les pièces le plus souvent disséquées, parfois injectées et annotées, doivent être lisibles, elles peuvent ainsi reposer sur une plaque de verre opaque, clair ou teinté (fig. 3). Dans la seconde moitié du $\mathrm{XX}^{\mathrm{e}}$ siècle, les collections réservées à la recherche sont conditionnées dans des contenants facilement manipulables, à la différence des préparations plus spécialement muséographiques. Ainsi, les dimensions du spécimen anatomique constituent-elles le seul et unique critère décisionnel quant au choix du contenant. Les encéphales de la collection du Dr. L. Palès (1905-1988), par exemple, sont conservés dans



Fig. 1. Série de flûtes présentant divers stades de développement de l'Homme. Le beau est ici associé au savant. Inv. A-8847; A-8848;A-8728;A-5072/1871-473. () MNHN/A. Verguin. 


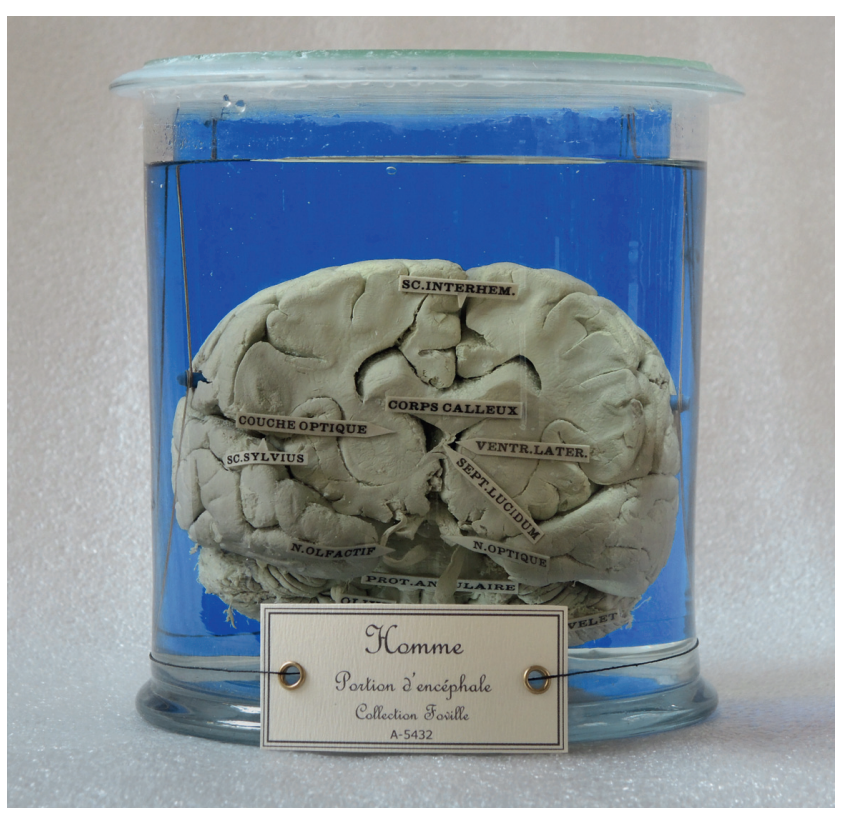

Fig. 2. Portion d'encéphale sur plaque bleue, disséqué par Foville, avec ses étiquettes anatomiques collées directement sur l'organe, qui confirment l'intérêt de ces restes humains pour la recherche, y compris contemporaine. Une série d'encéphale d'autres vertébrés préparés de la même façon était aussi présentée à des fins comparatives. Inv. A-5072. (C) MNHN/A. Verguin.

des cristallisoirs de dimensions identiques à ceux utilisés pour les encéphales de mammifères marins ou pour des spécimens entiers de taille comparable : le critère est donc bien dimensionnel.

Les restes humains conservés en fluides au MNHN sont des « individus entiers » ou des parties anatomiques choisies. Les spécimens entiers sont des séries d'embryons, de foetus et de nouveaux-nés qui ont documenté le thème du développement dans les différentes galeries successives d'Anatomie comparée ou d'Anthropologie. Une autre partie de la collection intéresse la tératologie et regroupe un grand nombre de pathologies rencontrées lors du développement. Ce thème se retrouve dans absolument toutes les collections médicales et vétérinaires en France, avant tout pour un propos de connaissances, même s'il a ensuite dérivé parfois vers des propos situés largement en dehors du champ de la science... Les pièces anatomiques isolées sont variables, et regroupent des têtes, des encéphales, des éléments d'appareils uro-génital, digestif, circulatoire ou respiratoire, etc.

Les restes humains en fluides ont, comme l'ensemble de la collection d'Anatomie comparée désormais, un statut de collection historique, puisque les plus récents datent de la première moitié $\mathrm{du} \mathrm{XX}^{\mathrm{e}}$ siècle, alors que les plus anciens (fond tératologique d'E. et I. Geoffroy St-Hilaire) ont été rassemblés dès le début du XIX ${ }^{\mathrm{e}}$ siècle. Cependant, cette relative ancienneté ne nuit en rien à l'intérêt scientifique de la collection. L'excellente qualité de la fixation et de la conservation fait que de nombreux projets actuels peuvent valoriser ces pièces dans différents domaines : scientifique, éducatif ou muséal.

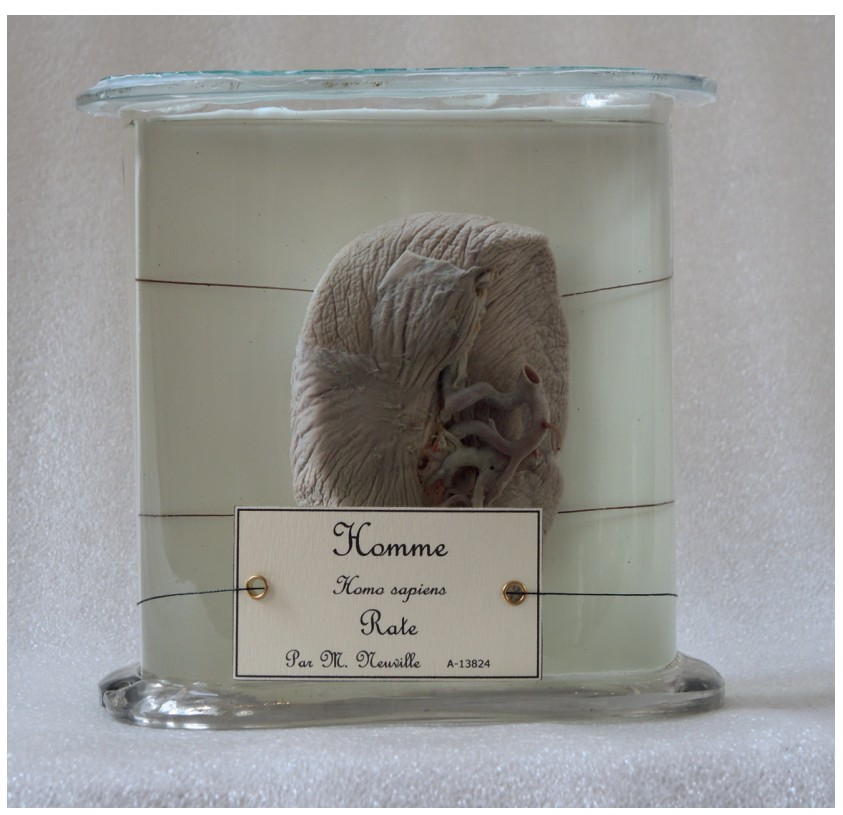

Fig. 3. Rate humaine injectée sur plaque blanche opaque. Cette pièce réalisée en novembre 1912 par Neuville était présentée pour montrer les différents viscères. Inv. A-13824/1912-100. () MNHN/A. Verguin.

\section{Éléments de gestion}

Les restes humains, de par leur constitution physiologique, ne diffèrent guère des restes des autres mammifères, et leur entretien n'est donc pas spécifique. Tout comme pour les collections en fluides en général, il faut donc veiller tout d'abord à la qualité des solutions de conservation, et faire en sorte que celles-ci soient compatibles avec les modalités de valorisation de la collection que l'on souhaite. La difficulté de gestion réside en la vérification de l'immersion constante des pièces ainsi qu'en la nécessité d'assurer, voire d'améliorer, les conditions d'étanchéité des contenants. Cependant, une pièce en partie émergée ne sera pas forcément immédiatement en péril, car le reste du corps immergé fonctionne comme une mèche et transmet tout de même une certaine quantité de conservateur dans les parties émergées. De plus, la partie émergée se trouve dans un environnement certes gazeux, mais contenant lui aussi des molécules de solution de conservation. Lorsque cela s'avère impératif, le renouvellement ou le changement de la solution de conservation doit être maîtrisé, car le fait de modifier la solution présente dans le contenant peut engendrer d'importantes altérations en matière de $\mathrm{pH}$ et de pression osmotique. Ainsi, le changement de pression osmotique peut-il provoquer soit le gonflement, soit, à l'inverse, la rétraction des tissus, ce qui entraîne immanquablement la rupture de ces tissus par tension mécanique ${ }^{5,6}$. Par conséquent, si la solution initiale n'est ni contaminée ni salie, on pourra se poser la question de savoir si on complète simplement la solution initiale ou si on la remplace complètement ${ }^{7}$. En effet, la plupart du temps, le spécimen a atteint un équilibre dans son milieu et un renouvellement complet peut potentiellement rompre cet équilibre et lui être dommageable. Une mesure 
$\mathrm{du} \mathrm{pH}$ et une estimation de la concentration de l'ancienne solution seront fort utile dans tous les cas de figure. La solution de conservation finale devra être neutre ou légèrement acide (entre 7,5 et 6), mais surtout tamponnée ${ }^{8}$. Ensuite, il faudra s'assurer que le contenant une fois lavé, et le milieu de conservation actualisé, soit bien correctement fermé (étanche à l'air, donc à l'oxygène et aux contaminants), avant de le remettre à sa place.

La gestion se fait selon le mode de valorisation de la collection. Il est en effet évident que les modalités de gestion ne peuvent être les mêmes selon que l'on destine la collection à une exposition permanente, à une itinérance d'exposition temporaire, à des valorisations pédagogiques ou à une approche de recherche fondamentale ou appliquée, pour ne citer que les modalités les plus courantes. Le mode de préparation, pour commencer, ne sera pas la même, le mode de marquage non plus, etc. Il faut garder en tête, comme pour toute collection de naturalia, y compris anthropologique, que chaque échantillon peut toujours, au fil du temps, servir de support de recherches et d'études.

Ce fait inné ne doit pas éradiquer les possibilités de valorisation avec intervention directe (dissection, prélèvement, etc.), mais doit au contraire motiver, de la part des gestionnaires, une parfaite connaissance des collections afin de pouvoir émettre une décision en toute connaissance de cause. Cela semble logique, mais la protection des collections et leur transmission à travers le temps ne peuvent s'accomplir qu'à ce prix. Quels seraient le sens et la justification de collections que l'on devrait uniquement regarder de loin? Une collection, y compris de restes humains, ne peut être considérée comme vivante qu'à partir du moment où elle est mise à disposition de communautés intellectuelles ou sociales, dans le respect des réglementations qui lui sont propres (loi «Musées de France », 2002, dites de bioéthique du 29 juillet 1994).

\section{Déontologie}

Mais si la gestion concerne le matériel et la manière de préserver, certaines pratiques plus spécialement universitaires ou scientifiques en général ont pu choquer, telles le stockage ou la présentation de restes humains mélangés avec des parties anatomiques animales ${ }^{9}$. Au-delà de la complexité des lois régissant le corps humain, il importe de garder en tête que notre époque ne comprend plus suffisamment la démarche scientifique au point de laisser de tels « mélanges » au fil des étagères. Les restes humains peuvent parfaitement servir d'archives scientifiques, voire même muséales, mais leur gestion réclame une attention qui ne soit pas uniquement matérielle. Des espaces dédiés seraient l'idéal, même si les locaux n’ont initialement pas été prévus pour cela.

\section{Conclusion}

Les collections de restes humains en fluides du MNHN de Paris sont un héritage des médecins/anatomistes dont certains se sont illustrés brillamment, lesquels ont légué à la postérité scientifique des pièces inestimables de par leur nature et leur richesse. $\mathrm{Au} \mathrm{XXI}{ }^{\mathrm{e}}$ siècle, cette collection historique sert encore de support à de nombreux développements scientifiques, éducatifs et muséaux. La plus grande partie de ces restes a été conservée pour cela et regroupée dans ce but. Ne nous privons pas de ce patrimoine exceptionnel, au risque de perdre une partie de la mémoire des collections, de la connaissance du corps humain, des savants et chercheurs qui ont fait progresser le savoir, et surtout, de ceux et de celles qui ont un jour décidé de donner leur corps pour le progrès. En ce sens, les collections de restes humains s'inscrivent profondément dans un devoir de mémoire. À nous désormais de l'accomplir.

\section{Notes}

1. Simmons, 2014

2. Neuville, 1917.

3. Cadot, 2007.

4. Crémière, 2004.

5. Steedman, 1976

6. Simmons, 1991

7. Herbin, 2013.

8. Herbin, 2012. 2008.

9. Symposium du musée du quai Branly,

\section{Bibliographie}

Cadot L., 2007, « Les restes humains: une gageure pour les musées? ", La lettre de l'OCIM, 109, p. 4-15.

Crémière C., 2004, La science au musée: L'anatomie comparée au jardin du Roi puis au Muséum d'histoire naturelle de Paris, 1745-1898, Thèse de doctorat, MNHN de Paris.
Herbin M., 2012, « La conservation des collections en fluide : quid du devenir de ces collections ", Biologie Géologie, 3, p. 179-188.

Herbin, M., 2013, « La conservation des collections en fluide : Approche historique et conservatoire ", Ceroart, [En ligne], I 2013, mis en ligne le 18 août 2013, consulté le 20 avril 2016. URL : http:// ceroart.revues.org/3432.

Neuville H., 1917, «Quelques remarques sur la formaldéhyde et son emploi ", Bulletin du Muséum national d'histoire naturelle de Paris, 2, p. 58-77.

Simmons J. E., 1991, “Conservation problems of fluid preserved collections", dans Natural History Museums: Direction of growth, Cato P.S. and C. Jones, Texas Tech. Univ. Press, Lubbock.

Simmons J.E., 2014, Fluid preservation: A comprehensive reference, Rowman \& Littlefield, Lanham, Boulder, New York, Toronto, Plymouth UK.

Steedman H.E., 1976, "General and applied data on formaldehyde fixation and preservation of marine zooplankton", dans Zooplankton Fixation and Preservation, H.F. Steedman, The UNESCO Press, Paris, France, p. 103-154.

Symposium du musée du quai Branly, 2008, «Des collections anatomiques aux objets de culte : conservation et exposition des restes humains dans les musées [en ligne] ", Actes du colloque, musée du quai Branly, 22-23 février 2008. http://www.quaibranly.fr/

\section{Sitographie}

www.legifrance.gouv.fr/affichTexte.do?cidT exte=JORFTEXT000000769536

www.legifrance.gouv.fr/affichTexte.do?cidT exte=JORFTEXT000000549618

www.legifrance.gouv.fr/affichTexte.do?cidT exte $=$ JORFTEXT000000549619\&categ orieLien $=$ id 